\title{
Clinical analysis of pain after transvaginal mesh surgery in patients with pelvic organ prolapse
}

\author{
Chang Shi, Ying Zhao, Qing Hu, Runqi Gong, Yitong Yin and Zhijun Xia* (1)
}

\begin{abstract}
Background: The purpose of this study was to investigate the relevant factors of pain after transvaginal mesh (TVM) surgery for the treatment of pelvic organ prolapse and to analyse the management and relief of the pain.

Methods: A multicentre retrospective study of a clinical database of patients who underwent TVM surgery was conducted, and pain related aspects were analysed.

Results: A total of 1855 patients were included in the study. We divided the patients into two groups: pain-free (1805 patients) and pain (50 patients) group. The incidence of pain after TVM surgery was $2.70 \%$, with a median occurrence time of 7.5 months. Pain mainly involved the vagina, perineum, buttocks, groin, inner thighs, and lower abdomen. Excessive intraoperative blood loss $(\mathrm{OR}=1.284,95 \% \mathrm{Cl} 0.868-2.401)$ and postoperative anatomic failure $(\mathrm{OR}=1.577$, 95\% Cl 0.952-3.104) were analysed as risk factors with statistical significance. Mesh exposure rate in the pain group was 38\%, showing a significant difference between the groups $(P<0.01)$. Forty patients underwent non-surgical treatment, with a relief rate of $40.0 \%, 33$ patients received surgical treatment, 15 underwent partial mesh removal, and 18 underwent complete mesh removal, with a relief rate of $84.8 \%$. The total relief rate was $88 \%$ within all 50 patients suffering from pain.
\end{abstract}

Conclusions: Excessive intraoperative bleeding and unsatisfactory postoperative anatomic outcomes can increase the risk of postoperative pain; mesh exposure is also associated with the pain. Most patients can get pain relief with proper management, more than half of whom may need mesh removal with differing approach.

Keywords: Transvaginal mesh surgery, Mesh complications, Postoperative pain, Pelvic organ prolapse

\section{Background}

Pelvic organ prolapse (POP) is a common pelvic floor disorder among women, with population-based epidemiologic studies reporting the prevalence range from 2.9 to $34.3 \%$ in general population [1-4]. Women have an $11-12.6 \%$ lifetime risk of surgery for POP by the age of 80 $[5,6]$. Traditional surgical procedures using weak native tissues have a high risk of failure, with almost $30 \%$ of the

\footnotetext{
*Correspondence: xiazj_tg@163.com

Department of Gynecology and Obstetrics, Shengjing Hospital of China Medical University, No. 36, Sanhao Street, Heping District, Shenyang 110004, Liaoning, China
}

patients requiring reoperation [7]. Transvaginal mesh (TVM) surgery, as a minimally invasive surgery, seems to provide better anatomic outcomes and appears to be an attractive option to treat POP $[8,9]$.

Although these devices have improved outcomes, the safety of synthetic mesh has been questioned owing to the surgical complications, prompting the US Food and Drug Administration to issue warnings about adverse events associated with the mesh [10]. Complications following TVM surgery include exposure, pain, sexual dysfunction, recurrent POP, and urogenital and rectovaginal fistulas [11]. Previous studies have reported a $1-3 \%$ 
incidence of pain after the pelvic floor repair procedures with mesh kits $[8,12]$. As one of the main complaints and complications, pain adversely affects a patient's quality of life to a great extent $[13,14]$. The risk factors and development mechanisms of the pain have not been fully understood; relevant factors reported include patient's overall health and oestrogen status, mesh materials, surgeon's experience, infection, and pelvic floor muscle spasms [15-17].

The primary objective of this clinical analysis, therefore, was to identify patient and surgical factors associated with the development of pain after the TVM surgery. The secondary aim was to conduct a clinical analysis of the management and relief of the pain.

\section{Methods}

In this multicentre retrospective study, patients who underwent TVM surgery for POP without hysterectomy were identified from the Pelvic Floor Medical Alliance of Northeast China and Inner Mongolia between January 2013 and October 2018. Patients were excluded if they had a history of chronic pain caused by endometriosis, vulvodynia, vaginismus, interstitial cystitis, or lower back conditions, since they have a higher risk of persistent postoperative pain and may not be ideal candidates for the synthetic material placement [18].

Operations were carried out by experienced urogynaecology surgeons. Relevant demographic characteristics and surgical data were extracted from patient electronic medical records. POP stage examinations were performed before and at 3 months follow-up after the surgery with the patient in a lithotomy position, according to the International Urogynecological Association (IUGA)/ International Continence Society (ICS) Pelvic Organ Prolapse Quantification (POP-Q) system [19]. Objective anatomic failure was defined if any point was at stage II or beyond, according to the POP-Q, which was in accordance with previous reports [20,21].

Patients were recommended to be followed-up at 3 months postoperatively and every 3-6 months subsequently. Follow-up included evaluating anatomic outcomes, screening for mesh exposure or erosion by physical examination, and inquiring about complications such as pain, vaginal discharge, vaginal bleeding, and sexual dysfunction. The IUGA/ICS joint terminology and classification was used to assess the postoperative complications [22]. Patients who met the criteria for category 1B-3B (provoked pain, pain during sexual intercourse, pain during physical activities, and spontaneous pain) were classified into a pain group, and the patients who did not meet the aforementioned criteria were classified into a pain-free group. For the pain group, detailed descriptions from the patients' medical records were collected, including pain occurrence time, type, location, degree, and remission. Pain caused by an intraoperative puncture injury and surgical incision is usually relieved in a short term after surgery without intervention, and was therefore not considered a complication.

The primary treatment of choice was non-surgical, including topical oestrogen, antibiotics, 1:5000 potassium permanganate sitz bath, biofeedback therapy, or a combination of the above. Patients in whom the conservative treatment was ineffective or who had the indications for mesh removal underwent surgery. Partial mesh removal was performed in cases of limited mesh exposure with no other bothersome symptoms and when mesh contracture was found. After irrigating with saline solution, we made an incision in the exposure or contracture site, removed the involved part of the mesh, and trimmed the edges of the vaginal epithelium. When the exposures were larger, presenting with severe symptoms, or mesh arms pierced the obturator space or ischiorectal fossa, the mesh was removed as much as possible. Complete mesh removal was performed by making an incision in the vaginal epithelium, dissecting the mesh from the overlying epithelium and underlying connective tissue, and closing the vagina with an absorbable suture. After the mesh and its arms were completely removed, a concomitant prolapse repair would be performed if needed. In cases of anatomic failure with obvious pain, we partially removed the mesh and reconnected the remaining part or performed complete mesh removal and reconstructed the normal anatomic structure with other repair procedures.

\section{Sample size calculation}

The sample size calculation was conducted by Power Analysis and Sample Size Software version 15.0 for Windows. According to statistics, 2260 patients underwent TVM surgery in a specified period. Considering problems such as incomplete data and loss of follow-up, the actual number of patients included in the study was about $80 \%$ of 2260 patients $(1,808)$. According to earlier studies, the incidence of pain is about $2 \%(1-3 \%)[8$, $12]$. When the sample proportion is 0.02 , a sample size of 1808 produces a two-sided $95 \%$ confidence interval (1-Alpha) with a width equal to 0.014 .

\section{Statistical analysis}

SPSS version 25.0 (IBM Statistics for Windows) was used for data analyses. Continuous variables are presented as the mean and standard deviation or median and ranges; categorical variables are summarised using number count and percentage. Independent samples t-test was used to compare continuous variables, chi-square or Fisher's exact test was used to compare categorical variables, and Mann-Whitney U test was used to compare 
rank variables between the groups. For variables with statistical differences, logistic regression was used to determine the contributions of the indicators to increase the risk of pain. A $P$ value $<0.05$ was considered statistically significant.

\section{Results}

A total of 2260 patients underwent TVM surgery for POP between January 2013 and October 2018. After the exclusion of 285 patients lost to follow-up, 97 with incomplete data, and 23 with baseline self-reported chronic pain, 1855 patients were included in this study. The median follow-up length with interquartile range was $24(11,31)$ months. Fifty $(2.7 \%)$ patients reported postoperative pain and were classified into the pain group, and the other 1805 patients were classified into the pain-free group.

A description of baseline demographic characteristics and surgical data of both groups are shown in Table 1. All patients had menopause. The mean age was $64.97 \pm 8.98$ years, and patients in the pain group were older than those in the pain-free group $(67.00 \pm 4.85 \mathrm{vs}$ $64.91 \pm 9.07$ years, $P=0.005)$. Differences in body mass index, parity, previous hysterectomy, previous POP surgery (without mesh implantation), and comorbidities including hypertension and diabetes mellitus were proportionally minor between the groups. Concomitant mid-urethral slings (MUS) for the treatment of stress urinary incontinence (SUI) were implanted in 244 (13.2\%) patients, and no differences were noted between the two groups in concomitant MUS or surgical procedure. Moreover, patients in the pain group were likely to lose more blood during the operation $(89.50 \pm 17.85$ vs $83.12 \pm 11.05 \mathrm{ml}, P=0.015$ ).

The POP-Q stage and values (Aa, Ba, Ap, Bp, C, D, and $\mathrm{Tvl}$ ) of both groups before and at 3 months after the surgery are presented in Tables 2 and 3. All patients had symptoms with grades III-IV POP preoperatively, with sufficient indications for prolapse surgery deemed by their surgeons. We found no difference in preoperative POP-Q evaluation between the two groups; whereas the differences of POP-Q stage $(P=0.038)$ and values of Aa, Ba, $C(P=0.019, P=0.029, P=0.016$, respectively $)$ at 3 months postoperatively were significant. Thirty patients in the pain-free group and four patients in the pain group experienced anatomic failure with the stage II.

Logistic analysis was performed on the contribution of each indicator to the risk of pain, including age, POP-Q values, and intraoperative blood loss. The combined effects of $\mathrm{Aa}, \mathrm{Ba}$, and $\mathrm{C}$ points were adopted for the assessment. The results showed that patients with greater blood loss $(\mathrm{OR}=1.28,95 \%$ CI $0.87-2.40, P=0.026)$ and lager POP-Q values $(\mathrm{OR}=1.58,95 \% \mathrm{CI} 0.95-3.10$, $P=0.011)$ had a higher risk of suffering from pain, while age was not statistically significant $(P=0.146)$.

The period between mesh implantation and pain occurrence ranged from 1 to 31 months, with a median of 7.5 months. The pain was localised in the vagina, perineum, buttocks, groin, lower abdomen, or multiple sites

Table 1 Patients characteristics and surgical data

\begin{tabular}{|c|c|c|c|c|c|c|c|}
\hline Characteristics & Total $(n=1855)$ & Pain $(n=50)$ & Pain-free $(n=1805)$ & $\begin{array}{l}\text { Mean } \\
\text { difference/ } \\
\text { OR }\end{array}$ & $95 \% \mathrm{Cl}$ & $t / x^{2}$ value & $P$ value \\
\hline Age, years & $64.97 \pm 8.98$ & $67.00 \pm 4.85$ & $64.91 \pm 9.07$ & 2.09 & 0.65 to 3.53 & 2.91 & $0.005^{*}$ \\
\hline $\mathrm{BMI}, \mathrm{kg} / \mathrm{m}^{2}$ & $23.66 \pm 1.79$ & $23.98 \pm 1.16$ & $23.65 \pm 1.80$ & 0.33 & -0.01 to 0.67 & 1.96 & 0.055 \\
\hline Parity, n & $2.27 \pm 0.90$ & $2.42 \pm 0.73$ & $2.27 \pm 0.90$ & 0.15 & -0.10 to 0.40 & 1.18 & 0.238 \\
\hline Hypertension & $1150(61.99)$ & $32(64.00)$ & $1118(61.94)$ & 1.09 & 0.61 to 1.96 & 0.09 & 0.767 \\
\hline Diabetes mellitus & $274(14.77)$ & $8(16.00)$ & $266(14.74)$ & 1.10 & 0.51 to 2.37 & 0.06 & 0.804 \\
\hline Previous hysterectomy & $51(2.75)$ & $2(4.00)$ & $49(2.71)$ & 1.49 & 0.35 to 6.32 & 0.30 & 0.646 \\
\hline Previous POP surgery & $376(20.27)$ & $9(18.00)$ & $367(20.33)$ & 0.86 & 0.41 to 1.79 & 0.16 & 0.686 \\
\hline \multicolumn{8}{|l|}{ Surgical procedure } \\
\hline Total & $1641(88.46)$ & $46(92.00)$ & $1595(88.36)$ & 1.51 & 0.54 to 4.25 & 0.66 & 0.935 \\
\hline Anterior & $63(3.40)$ & $1(2.00)$ & $62(3.43)$ & 0.57 & 0.08 to 4.22 & & \\
\hline Posterior & $151(8.14)$ & $3(6.00)$ & $148(8.20)$ & 0.72 & 0.22 to 2.32 & & \\
\hline Concomitant MUS surgery & $244(13.15)$ & $7(14.00)$ & $237(13.13)$ & 1.08 & 0.48 to 2.42 & 0.03 & 0.858 \\
\hline Operation time, min & $78.73 \pm 11.41$ & $81.50 \pm 12.76$ & $78.65 \pm 11.37$ & 2.85 & -0.36 to 6.06 & 1.74 & 0.082 \\
\hline Estimated blood lose, ml & $83.28 \pm 11.33$ & $89.50 \pm 17.85$ & $83.12 \pm 11.05$ & 5.38 & 1.28 to 11.48 & 2.51 & $0.015^{*}$ \\
\hline
\end{tabular}

Values are presented as mean $\pm S D$, median (range), and $\mathrm{n}$ (percentage)

$B M I$ body mass index, MUS mid-urethral slings

${ }^{*} P<0.05$ 


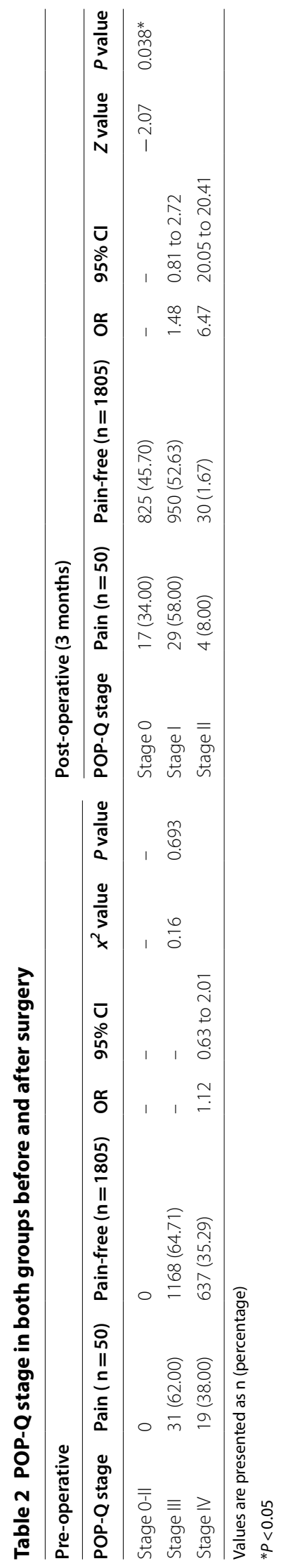




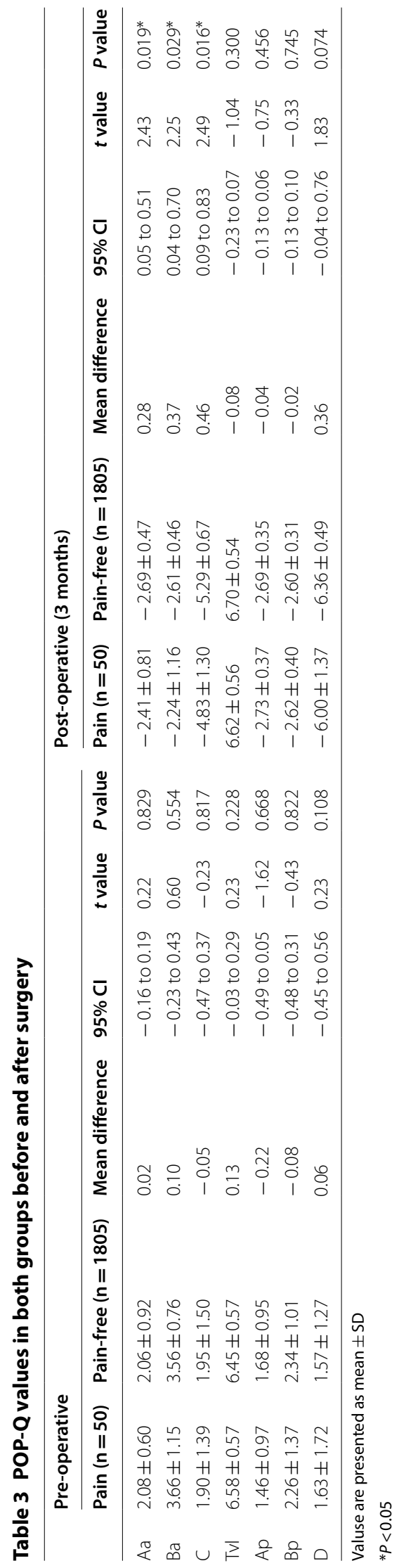


(Fig. 1). Most of the patients had spontaneous pain (88\%); pain in the buttocks, groin, and inner thigh was sometimes aggravated while sitting or by certain movements such as walking, urination, and defecation. Points of tenderness or cord-like changes could sometimes be found at the physical examination in patients with vaginal pain. Mesh exposure was found in 19 patients, 13 of whom experienced vaginal discharge or developed local inflammation. The exposure rate in the pain group was 38\% $(19 / 50)$ and the pain-free group was $4.4 \%$ (79/1805), with a significant difference between the groups $(\mathrm{OR}=13.39$, 95\% CI 7.25-24.74, $P<0.01$ ).

The management measures and pain remission are summarised in Table 4 and Fig. 1. A majority of patients $(80 \%)$ had received conservative treatment, including topical oestrogen, antibiotics, and physiotherapy; 33 patients, in whom the non-surgical management was unsuccessful or who had indications, received surgical intervention, including partial mesh removal for 15 patients and complete mesh removal for 18 patients. A total of $44(88 \%)$ patients reported pain relief after the management, 16 by conservative treatment and 28 by surgical intervention.

\section{Discussion}

In this retrospective study, we investigated the relevant factors of pain after TVM surgery for the treatment of POP, and analysed the management and relief of pain based on our clinical experience. The incidence of pain in our study was $2.7 \%$. Excessive intraoperative blood loss and large postoperative POP-Q values were considered as risk factors. The vagina was the most common site of pain, followed by the perineum, buttock, and groin; few patients had lower abdomen or generalised pain. In some patients, pain could be relieved by conservative therapy. Mesh removal was an effective treatment with the relief rate of $84.8 \%$, and mesh exposure was the most common reason for the removal.

The process by which pain develops after TVM surgery is probably multifactorial, and the manifestations of pain are varied. The relationship between excessive intraoperative blood loss and postoperative pain has not been determined. A previous study shows that excessive intraoperative blood loss can increase the risk of mesh exposure 7.3-fold [23] and may lead to postoperative inflammatory reaction, which may be related to the development of pain. One out five patients with mesh exposure in our study reported pain, compared with $0-54 \%$ in the previous studies $[13,18,20,24]$. The exposure itself may not have been the cause of pain, while the resulting inflammatory reaction might have been relevant. It has been proposed that chronic inflammatory response caused by the exposed mesh can lead to vaginal pain [25]. Unsatisfactory postoperative anatomic outcomes may also increase the risk of pain, which may be related to the insufficient tension of the mesh. Patients with this condition may experience abdominal and perineal distension accompanied by the pain, which can also occur occasionally in untreated POP patients. In addition, although MUS is also a transvaginal implant, the concomitant MUS implantation for SUI was thought to be unrelated to the pain, in accord with previous findings [26].

Pain can sometimes resolve on its own or improve with physiotherapy, oestrogen cream, or antibiotic treatment $[13,27]$. Since pain usually includes hypertonia of the pelvic floor muscles, pelvic floor physiotherapy has showed a good curative effect [28]. For the mesh exposure, more aggressive management might be required when the pain appears, rather than conservative treatment such as topical oestrogen and closure of the vaginal epithelium [27]. Partial or total mesh removal is a better option for patients who have not responded to the conservative treatment, and the rate of pain relief after the mesh removal ranges from 50 to $84 \%$ in prior studies [18].

Pain can also result from mesh arms piercing the obturator space or ischiorectal fossa [29, 30], which can be markedly improved after the mesh is removed. Thus, during the process of puncture for mesh implantation, we would like to emphasise that the implants must be placed in the interstitial space rather than in the tissue. In some cases, pain can be explained by bunching, folding, or contracture of the mesh $[17,29]$. Mesh contracture may result in a concomitant contracture of the underlying pelvic floor musculature and excessive tension on the mesh arms, causing increased pelvic floor muscle tone and tenderness $[17,30]$. Pain in these cases usually is unresponsive to conservative measures, but can be relieved following mesh removal. In addition, some patients had persistent pain from the moment the mesh was implanted, while no abnormality was found at the examination. In a previous study, the removal of all vaginally accessible meshes was performed in such cases [18]. Both conservative and surgical approaches in our study were used, while the improvement was unsatisfactory. The mechanism of such pain has not been clearly identified. Nevertheless, new complications may be associated with the removal of the mesh, including recurrent POP, and a concomitant prolapse repair should be performed if needed.

In this study, a small number of patients had undergone previous hysterectomy. Although several approaches including TVM for the management of POP have been reported, the best strategy for posthysterectomy vaginal vault prolapse (VVP) remains 


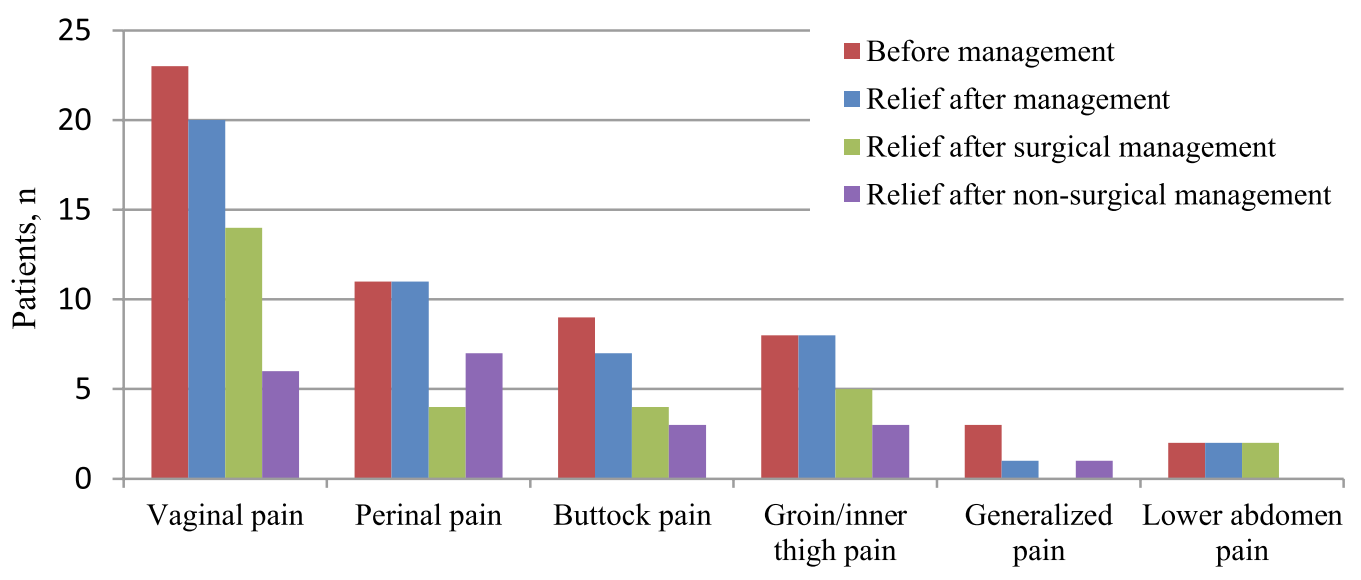

Fig. 1 Sites of the pain and relief after treatment

Table 4 Managements for patients in pain group

\begin{tabular}{lll}
\hline Precedure & Total & Relief \\
\hline Conservative treatment & 40 & 16 \\
Topical estrogen & 32 & - \\
Antibiotics & 11 & - \\
Physical therapy & 35 & - \\
Surgical intervention & 33 & 28 \\
Partial mesh removal & 15 & 12 \\
Mesh exposure & 6 & 5 \\
Mesh contracture/folding & 6 & 6 \\
Mesh arms piercing obturator space/ischiorectal fossa & 1 & 0 \\
Anatomy failure & 2 & 1 \\
Complete mesh removal & 18 & 16 \\
Mesh exposure & 7 & 7 \\
Mesh arms piercing obturator space/ischiorectal fossa & 8 & 7 \\
Anatomy failure & 2 & 2 \\
No abnormality on examination & 1 & 0 \\
\hline
\end{tabular}

controversial. Studies have suggested that laparoscopic sacrocolpopexy and sacrospinous fixation in the treatment of primary VVP and transvaginal bilateral sacrospinous fixation in the treatment of recurrent VVP appears to be effective and safe for the improvement of quality of life and sexual function [31]. As noted in a systematic review, TVM surgery had the highest reoperation rates (including complications and recurrence) in the treatment of VVP [32]. Therefore, we should be more careful in evaluating and selecting the appropriate approach before the TVM is intended for the reconstruction of VVP.

Health-related quality of life (HR-QoL) is widely recognised as an important outcome measure following urogynaecological surgery [33]. Taking into account the significant impact of POP on physical and mental health [34], in addition to the surgical effect, that is, anatomical reduction, surgeons should focus on the assessment of postoperative function recovery and improvement of quality of life of the patients. Therefore, multidisciplinary approach in the treatment of women with POP is very important. Although studies have shown that general HR-QoL improved significantly (mainly shown as improvement of sexual activity, mobility, excretion, depression, etc.) after apical POP reconstructive surgery [35], the use of TVM can still lead to some serious complications, such as pain, sexual dysfunction, and mesh exposure [11]. Therefore, the application of TVM should have strict indications, and is usually recommended for patients $\geq 50$ years old or with low sexual activity, severe pelvic floor structural damage, or recurrence [21,34]. Surgeons should weigh the risks and benefits on an individual level based on the patient characteristics when opting for mesh kits for surgical repair, to achieve better treatment outcomes in patients with POP.

This study had the following limitations: Firstly, pain was not directly measured with validated instruments, and the metric used for pain relief was based on subjective phrases in medical records at times. Secondly, since the mesh we used was mainly Prolift ${ }^{\mathrm{TM}}$, the factors of mesh itself (size, shape and material) are not included in the study. Then, since the pain may occur years after surgery, longer-term follow-up might be required. Finally, the cases in this study came from specific regions, and it is unclear whether the conclusions can apply to patients in other parts of the world. This study had several limitations. First, pain was not directly measured with validated instruments, and the metric used for analysis of pain relief was based on subjective phrases in medical records 
at times. Second, since the pain may occur years after the surgery, longer-term follow-up might be required. Third, the cases in this study were collected from specific geographical regions, and it is unclear whether the conclusions can apply to patients in other parts of the world.

Despite these limitations, the evidence of our study is strengthened by the relatively large number of patients and detailed data available from surgical and medical records. Moreover, this study investigated the relevant factors of postoperative pain, one of the inconvenient complications after the TVM surgery, the observations of which are relatively new to the available literature. The clinical practice shown in this study may provide important information about the strategy of the pain management following TVM surgery. In the future, we will carry out more in-depth studies on the mesh complications to provide more important reference for the postoperative management of TVM.

\section{Conclusions}

The aetiology and development of pain after TVM surgery involves a variety of factors. Excessive intraoperative bleeding and failure with postoperative anatomic outcomes can increase the risk of pain, and patients with mesh exposure are more likely to develop this symptom. Patients can obtain pain relief with non-surgical or surgical management, more than half of whom need mesh removal with differing approach. More factors related to the postoperative pain remain to be explored. Through continuous research and improvement, we hope that TVM surgery can have better effects and safety.

\section{Abbreviations}

POP: Pelvic organ prolapse; TVM: Transvaginal mesh; POP-Q: Pelvic organ prolapse quantification; SUI: Stress urinary incontinence; MUS: Mid-urethral slings; WP: Vaginal vault prolapse; HR-QoL: Health-related quality of life.

\section{Acknowledgements}

Not applicable.

\section{Authors' contributions}

CS: study design, data analysis, manuscript writing; $Y Z$ : data collection and analysis; $\mathrm{QH}$ : project development, data collection; $\mathrm{RG}, \mathrm{YY}$ : critical revision of the manuscript; ZX: Study design, project development, manuscript writing. All authors read and approved the final manuscript.

\section{Funding}

This work was supported by Shenyang Science and Technology Plan Project (18-014-4-29). The funder gave us financial help to conduct the research but was not involved in study design, data collection, analysis, interpretation or in the writing of the report. The study's content is solely the responsibility of the authors.

\section{Availability of data and materials}

The datasets generated and/or analyzed during the current study are not publicly available due to ethical issues and potential for organizational privacy to be compromised, but are available from the corresponding author upon reasonable request.

\section{Ethics approval and consent to participate}

The Ethics Committee of Shengjing Hospital of China Medical University approved this retrospective study, and granted the permission to access and use the medical records described in the study (No. 2019PS482K). As the study was based on data routinely collected from electronic medical records, no additional testing/inspections were needed for patients who involved in the research, the Ethics Committee approved the waiver of informed consent.

\section{Consent to publication}

Not applicable.

\section{Competing interests}

The authors declare that they have no competing interests.

Received: 2 July 2020 Accepted: 21 January 2021

Published online: 30 January 2021

\section{References}

1. Hendrix SL, Clark A, Nygaard I, Aragaki A, Barnabei V, McTiernan A. Pelvic organ prolapse in the Women's Health Initiative: gravity and gravidity. Am J Obstet Gynecol. 2002;186(6):1160-6.

2. Handa VL, Garrett E, Hendrix S, Gold E, Robbins J. Progression and remission of pelvic organ prolapse: a longitudinal study of menopausal women. Am J Obstet Gynecol. 2004;190(1):27-32.

3. Nygaard I, Barber MD, Burgio KL, et al. Prevalence of symptomatic pelvic floor disorders in US women. JAMA J Am Med Assoc. 2008;300(11):1311-6.

4. Slieker-ten Hove MCP, Pool-Goudzwaard AL, Eijkemans MJC, SteegersTheunissen RPM, Burger CW, Vierhout ME. The prevalence of pelvic organ prolapse symptoms and signs and their relation with bladder and bowel disorders in a general female population. Int Urogynecol J. 2009;20(9):1037-45.

5. Olsen AL, Smith VJ, Bergstrom JO, Colling JC, Clark AL. Epidemiology of surgically managed pelvic organ prolapse and urinary incontinence. Obstet Gynecol. 1997:89(4):501-6.

6. Wu JM, Matthews CA, Conover MM, Pate V, Funk MJ. Lifetime risk of stress urinary incontinence or pelvic organ prolapse surgery. Obstet Gynecol. 2014;123(6):1201-6.

7. Barber MD, Maher C. Epidemiology and outcome assessment of pelvic organ prolapse. Int Urogynecol J. 2013;24(11):1783-90.

8. Diwadkar GB, Barber MD, Feiner B, Maher C, Jelovsek JE. Complication and reoperation rates after apical vaginal prolapse surgical repair a systematic review. Obstet Gynecol. 2009;113(2):367-73.

9. Maher C, Feiner B, Baessler K, Christmann-Schmid C, Haya N, Marjoribanks J. Transvaginal mesh or grafts compared with native tissue repair for vaginal prolapse. Cochrane Database Syst Rev. 2016;2(2):Cd 012079.

10. US Food and Drug Administration. FDA public health notification: serious complications associated with transvaginal placement of surgical mesh in repair of pelvic organ prolapse and stress urinary incontinence. FDA. gov. 2008. http://www.fda.gov/MedicalDevices/Safety/AlertsandNotices/ PublicHealthNotifications/ucm061976.htm. Accessed 17 Apr 2016.

11. Firoozi F, Ingber MS, Moore CK, Vasavada SP, Rackley RR, Goldman HB. Purely transvaginal/perineal management of complications from commercial prolapse kits using new prostheses/grafts complication classification system. J Urol. 2012;187(5):1674-9.

12. Feiner B, Jelovsek JE, Maher C. Efficacy and safety of transvaginal mesh kits in the treatment of prolapse of the vaginal apex: a systematic review. Br J Obstet Gynaecol. 2009;116(5):15-24.

13. Tijdink MM, Vierhout ME, Heesakkers JP, Withagen MIJ. Surgical management of mesh-related complications after prior pelvic floor reconstructive surgery with mesh. Int Urogynecol J. 2011;22(11):1395-404.

14. Geller EJ, Babb E, Nackley AG, Zolnoun D. Incidence and risk factors for pelvic pain following mesh implant surgery for the treatment of pelvic floor disorders. J Minim Invas Gyn. 2007;24(1):67-73.

15. Nguyen JN, Jakus-Waldman SM, Walter AJ, White T, Menefee SA. Perioperative complications and reoperations after incontinence and prolapse surgeries using prosthetic implants. Obstet Gynecol. 2012;119(3):539-46. 
16. Barski D, Otto T, Gerullis H. Systematic review and classification of complications after anterior, posterior, apical, and total vaginal mesh implantation for prolapse repair. Surg Tech Int. 2014;24(24):217-24

17. Gyang AN, Feranec JB, Patel RC, Lamvu GM. Managing chronic pelvic pain following reconstructive pelvic surgery with transvaginal mesh. Int Urogynecol J. 2014;25(3):313-8.

18. Crosby EC, Abernethy M, Berger MB, DeLancey JO, Fenner DE, Morgan DM. Symptom resolution after operative management of complications from transvaginal mesh. Obstet Gynecol. 2014;123(1):134-9.

19. Haylen BT, Maher CF, Barber MD, et al. An International Urogynecological Association (IUGA)/International Continence Society (ICS) joint report on the terminology for female pelvic organ prolapse (POP). Neurourol Urodyn. 2016;35(2):137-68.

20. Nieminen $K$, Hiltunen $R$, Takala T, et al. Outcomes after anterior vaginal wall repair with mesh: a randomized, controlled trial with a 3 year followup. Am J Obstet Gynecol. 2010;203(3):235.e1.

21. Kinjo M, Yoshimura Y, Kitagawa Y, Okegawa T, Nutahara K. Sexual activity and quality of life in Japanese pelvic organ prolapse patients after transvaginal mesh surgery. J Obstet Gynaecol Re. 2018;44(7):1302-7.

22. Haylen BT, Freeman RM, Swift SE, et al. An International Urogynecological Association (IUGA)/International Continence Society (ICS) joint terminology and classification of the complications related directly to the insertion of prostheses (meshes, implants, tapes) and grafts in female pelvic floor surgery. Neurourol Urodyn. 2011;30(1):2-12.

23. Gold KP, Ward RM, Zimmerman CW, et al. Factors associated with exposure of transvaginally placed polypropylene mesh for pelvic organ prolapse. Int Urogynecol J. 2012;23(10):1461-6.

24. Glazener CM, Breeman S, Elders A, et al. Mesh, graft, or standard repair for women having primary transvaginal anterior or posterior compartment prolapse surgery: two parallel-group, multicentre, randomised, controlled trials (PROSPECT). Lancet. 2017;389(10067):381-92.

25. Cundiff GW, Quinlan DJ, van Rensburg JA, Slack M. Foundation for an evidence-informed algorithm for treating pelvic floor mesh complications: a review. BJOG. 2018;125(8):1026-37.

26. Lee D, Dillon B, Lemack G, Gomelsky A, Zimmern P. Transvaginal mesh kits-how "serious" are the complications and are they reversible? Urology. 2013;81(1):43-8.
27. Jeffrey ST, Nieuwoudt A. Beyond the complications: medium-term anatomical, sexual and functional outcomes following removal of trocarguided transvaginal mesh. A retrospective cohort study. Int Urogynecol J. 2012;23(10):1391-6.

28. Rosenbaum TY, Owens A. The role of pelvic floor physical therapy in the treatment of pelvic and genital pain-related sexual dysfunction (CME). J Sex Med. 2008;5(3):513-23.

29. Marcus-Braun N, Bourret A, von Theobald P. Persistent pelvic pain following transvaginal mesh surgery: a cause for mesh removal. Eur J Obstet Gynecol Reprod Biol. 2012;162(2):224-8.

30. Feiner B, Maher C. Vaginal mesh contraction: definition, clinical presentation, and management. Obstet Gynecol. 2010;115(2):325-30.

31. Vitale SG, Lagana AS, Noventa M, et al. Transvaginal bilateral sacrospinous fixation after second recurrence of vaginal vault prolapse: efficacy and impact on quality of life and sexuality. Biomed Res Int. 2018;2018:1-6.

32. Coolen ALWM, Bui BN, Dietz V, et al. The treatment of post-hysterectomy vaginal vault prolapse: a systematic review and meta-analysis. Int Urogynecol J. 2018;28(12):1767-83.

33. Barber MD, Maher C. Apical prolapse. Int Urogynecol J. 2013:24(11):1815-33

34. Lagana AS, La Rosa VL, Rapisarda AMC, Vitale SG. Pelvic organ prolapse: the impact on quality of life and psychological well-being. J Psychosom Obst Gynecol. 2018;39(2):164-6.

35. Altman D, Geale K, Falconer C, Morcos E. A generic health-related quality of life instrument for assessing pelvic organ prolapse surgery: correlation with condition-specific outcome measures. Int Urogynecol J. 2018:29(8):1093-9.

\section{Publisher's Note}

Springer Nature remains neutral with regard to jurisdictional claims in published maps and institutional affiliations.
Ready to submit your research? Choose BMC and benefit from:

- fast, convenient online submission

- thorough peer review by experienced researchers in your field

- rapid publication on acceptance

- support for research data, including large and complex data types

- gold Open Access which fosters wider collaboration and increased citations

- maximum visibility for your research: over $100 \mathrm{M}$ website views per year

At $\mathrm{BMC}$, research is always in progress.

Learn more biomedcentral.com/submissions 